\title{
Anisotropic electronic, mechanical, and optical properties of monolayer $\mathrm{WTe}_{2}$
}

\author{
E. Torun, ${ }^{1, a)}$ H. Sahin, ${ }^{1}$ S. Cahangirov, ${ }^{2,3}$ A. Rubio, ${ }^{3,4}$ and F. M. Peeters ${ }^{1}$ \\ ${ }^{1}$ Department of Physics, University of Antwerp, Groenenborgerlaan 171, B-2020 Antwerp, Belgium \\ ${ }^{2}$ UNAM-National Nanotechnology Research Center and Institute of Materials Science and Nanotechnology, \\ Bilkent University, Ankara 06800, Turkey \\ ${ }^{3}$ Nano-Bio Spectroscopy Group and ETSF, Dpto. Física de Materiales, Universidad del País Vasco, \\ CFM CSIC-UPVIEHU-MPC \& DIPC, 20018 San Sebastián, Spain \\ ${ }^{4}$ Max Planck Institute for the Structure and Dynamics of Matter and Center for Free-Electron Laser Science, \\ Luruper Chaussee 149, 22761 Hamburg, Germany
}

(Received 4 November 2015; accepted 4 February 2016; published online 19 February 2016)

\begin{abstract}
Using first-principles calculations, we investigate the electronic, mechanical, and optical properties of monolayer $\mathrm{WTe}_{2}$. Atomic structure and ground state properties of monolayer $\mathrm{WTe}_{2}\left(\mathrm{~T}_{d}\right.$ phase $)$ are anisotropic which are in contrast to similar monolayer crystals of transition metal dichalcogenides, such as $\mathrm{MoS}_{2}, \mathrm{WS}_{2}, \mathrm{MoSe}_{2}, \mathrm{WSe}_{2}$, and $\mathrm{MoTe}_{2}$, which crystallize in the H-phase. We find that the Poisson ratio and the in-plane stiffness is direction dependent due to the symmetry breaking induced by the dimerization of the $\mathrm{W}$ atoms along one of the lattice directions of the compound. Since the semimetallic behavior of the $\mathrm{T}_{d}$ phase originates from this $\mathrm{W}-\mathrm{W}$ interaction (along the $a$ crystallographic direction), tensile strain along the dimer direction leads to a semimetal to semiconductor transition after $1 \%$ strain. By solving the Bethe-Salpeter equation on top of single shot $\mathrm{G}_{0} \mathrm{~W}_{0}$ calculations, we predict that the absorption spectrum of $\mathrm{T}_{d}-\mathrm{WTe}_{2}$ monolayer is strongly direction dependent and tunable by tensile strain. (C) 2016 AIP Publishing LLC.
\end{abstract}

[http://dx.doi.org/10.1063/1.4942162]

\section{INTRODUCTION}

Single layer transition metal dichalcogenides (TMDs) are promising candidates for next generation of flexible nanoelectronic devices due to their wide range of remarkable properties. ${ }^{1-3}$ The chemical formula of TMDs is $\mathrm{MX}_{2}$, where $\mathrm{M}$ stands for a transition metal (e.g., Mo and $\mathrm{W}$ ) and $\mathrm{X}$ is a chalcogen atom (e.g., S, Se, and Te). One of the most important properties of TMDs is the crossover from indirect to direct band gap when the number of layers is reduced to a single layer. ${ }^{4,5}$ Bulk TMDs are layered structures that are held together by weak van der Waals interaction. A single layer TMD can be obtained from their three-dimensional (3D) counterpart by using, e.g., the micromechanical cleavage technique or they can be synthesized by using chemical vapor deposition (CVD). Most of these materials are either in the trigonally coordinated $\mathrm{H}$ phase or the octahedrally coordinated $\mathrm{T}$ phase, and very few of them are stable in both $\mathrm{T}$ and $\mathrm{H}$ phases. Rarely some of them can be found in the $\mathrm{T}_{d}$ structure where there are bonds between the metal atoms so that they dimerize along one of the lattice directions. ${ }^{6,7}$ The stability of these phases is explained by the competing effects between ligand field splitting of the $d$-orbitals energy levels of the transition metals and the charge density wave instability together with structural phase transition. ${ }^{8}$

Although the atoms which form $\mathrm{WTe}_{2}$ are located in the same row of the periodic table as the compounds with $\mathrm{H}$ phase as their ground state, the ground state of $\mathrm{WTe}_{2}$ is the $\mathrm{T}_{d}$ structure. This difference in the geometric structure

${ }^{\text {a)} E l e c t r o n i c ~ m a i l: ~ e n g i n . t o r u n @ u a n t w e r p e n . b e ~}$ separates $\mathrm{WTe}_{2}$ from these $\mathrm{H}$-phase compounds. Earlier reports suggested that $\mathrm{T}_{d^{-}}-\mathrm{WTe}_{2}$ is a semimetallic compound, ${ }^{9-12}$ in contrast to other TMDs in the H-phase, i.e., $\mathrm{MoS}_{2}, \mathrm{MoSe}_{2}, \mathrm{WS}_{2}$, and $\mathrm{WSe}_{2}$ that are semiconductors. In addition to its semimetallic nature, very recent studies showed that $\mathrm{T}_{d}-\mathrm{WTe}_{2}$ has other remarkable properties, such as superconductivity and anisotropic magnetoresistance, which makes the compound quite attractive for nanoelectronics applications. ${ }^{13-26}$

Motivated by these observations, in this work, we investigate the anisotropic electronic, mechanical, and optical properties of monolayer $\mathrm{T}_{d}-\mathrm{WTe}_{2}$ using first-principle calculations. We found that (i) the mechanical properties such as Poisson's ratio and in-plane stiffness are strongly anisotropic, (ii) not only electronic properties are anisotropic but also strain tunable semimetal-to-semiconductor transition takes place even at low tensile strains, and (iii) the dielectric response of the structure along parallel and perpendicular directions to the W-W dimer displays significant differences.

This paper is organized as follows: Computational details are given in Sec. II, the discussion on the stability of the different phases, the electronic, mechanical, and optical properties of $\mathrm{WTe}_{2}$ monolayer are presented in Sec. III. Our results are concluded in Sec. IV.

\section{METHODOLOGY}

All calculations are performed using the projector augmented wave $(\mathrm{PAW})^{28,29}$ potentials as implemented in the Vienna Ab-initio Simulation Package (VASP) including spinorbit coupling (SOC).$^{30,31}$ The electronic exchange-correlation 
potential is treated within the polarized generalized gradient approximation (GGA) of Perdew-Burke-Ernzerhof (PBE). ${ }^{32}$ A plane-wave basis set with kinetic energy cutoff of $500 \mathrm{eV}$ is used. A vacuum spacing $\sim 11 \AA$ is taken to prevent layer-layer interactions. A set of $20 \times 10 \times 1 \Gamma$ centered k-point sampling is used for the primitive unit cell and scaled according to the sizes of the supercells. The convergence criterion for energy is set to $10^{-5} \mathrm{eV}$ between two consecutive steps in the self-consistent field calculations. The atomic positions are relaxed until the Hellmann-Feynman forces are less than $10^{-4} \mathrm{eV} / \AA$. Pressures on the lattice unit cell are decreased to values less than $1.0 \mathrm{kbar}$. The charge transfer between the atoms is calculated by using Bader's charge analysis. ${ }^{33}$

In order to investigate the anisotropic optical properties of monolayer $\mathrm{WTe}_{2}$, we performed a single shot GW calculation $\left(\mathrm{G}_{0} \mathrm{~W}_{0}\right)$ on top of the standard density functional theory (DFT) calculations including SOC. Then, we obtain the absorption spectrum by solving the Bethe-Salpeter equation (BSE) on top of the $\mathrm{G}_{0} \mathrm{~W}_{0}$ calculation. During this process, we used $6 \times 3 \times 1 \Gamma$ centered $\mathbf{k}$-point sampling for the rectangular $\mathrm{T}_{d}-\mathrm{WTe}_{2}$ unit cell. The cutoff for the response function was set to $200 \mathrm{eV}$. The number of bands used in our calculation is 160 . The cutoff energy for the plane-waves was chosen to be $400 \mathrm{eV}$. We include 4 valence and 4 conduction bands into the calculations in the BSE step. We checked the convergence of the absorption spectra with respect to number of bands and energy cutoff for the plane-waves.

\section{RESULTS}

\section{A. Stability of $\mathrm{WTe}_{2}$}

Earlier reports suggest that $\mathrm{WTe}_{2}$ is either in the $\mathrm{T}_{d}^{9-11,34}$ or the $\mathrm{H}^{27}$ phase. Both phases are shown in Fig. 1. In order to obtain the most favorable structure of the compound, we compared the total energies of the $2 \times 2$ unit cells of the $\mathrm{H}$ and $\mathrm{T}_{d}$ structures of $\mathrm{WTe}_{2}$. We found that the $\mathrm{T}_{d}$ structure is energetically the most favorable structure when in the monolayer form. The structure has $\sim 0.075 \mathrm{eV}$ lower energy per formula unit than the $\mathrm{H}$ structure, which is consistent with the earlier reported value. ${ }^{8}$ The $\mathrm{H}$ phase of the compound is a semiconductor while the $\mathrm{T}_{d}$ phase is semimetallic. The ground state properties of both phases are listed in Table I.

To examine the dynamic stability of the $\mathrm{H}$ and the $\mathrm{T}_{d}$ structures of $\mathrm{WTe}_{2}$, we calculated the phonon spectra. Phonon spectra are calculated using the small displacement method as implemented in the PHON software package. ${ }^{36}$ The force constant matrix is calculated by displacing atoms from their equilibrium positions in a $(6 \times 6 \times 1)$ and $(6 \times 3 \times 1)$ supercell for the $\mathrm{H}$ and the $\mathrm{T}_{d}$ structures, respectively. As seen in Fig. 4, the phonon spectra for both the $\mathrm{H}$ and $\mathrm{T}_{d}$ structure have no imaginary frequencies in the whole Brillouin zone (BZ) which indicates that there is a restoring force for any possible distortion around equilibrium. Small imaginary frequencies in the out-of-plane acoustic mode near the $\Gamma$ point are numerical artifacts caused by the inaccuracy of the FFT grid to account for the rapid decay in the out-of-plane force constants. The distortion in the $\mathrm{T}_{d}$ structure lifts certain degeneracies that are present in the $\mathrm{H}$ structure. There is a gap between the acoustic and optic phonon branches of the $\mathrm{H}$ structure while in the $\mathrm{T}_{d}$ structure this gap is closed and the acoustic and optic modes are hybridized. In addition, our analysis of the vibrational character of the eigenmodes reveals structural differences between these two phases of $\mathrm{WTe}_{2}$ : (i) $\mathrm{H}$ phase has three Raman-active modes at $117 \mathrm{~cm}^{-1}$, $173 \mathrm{~cm}^{-1}$, and $191 \mathrm{~cm}^{-1}$ that have $\mathrm{E}^{\prime \prime}, \mathrm{A}_{1}^{\prime}$, and $\mathrm{E}^{\prime}$ symmetries, respectively. (ii) $\mathrm{T}_{d}$ phase has many mixed eigenmodes of inplane and out-of-plane vibrations including two characteristic Raman-active modes at $149 \mathrm{~cm}^{-1}$ and $234 \mathrm{~cm}^{-1}$. The lowfrequency one corresponds to an $\mathrm{E}_{\mathrm{g}}$-like in-plane counterphase motion of $\mathrm{W}$ and $\mathrm{Te}$ atoms while the high-frequency phonon branch corresponds to an out-of-plane counter-phase motion of the $\mathrm{W}$ and the Te atoms. The presence of these distinctive phonon modes in the two phases allows to distinguish between the two phases $\mathrm{WTe}_{2}$ via Raman measurements.

\section{B. Mechanical properties}

As mentioned before, the $\mathrm{T}_{d}$ phase of monolayer $\mathrm{WTe}_{2}$ is more stable than its $\mathrm{H}$ phase (by $30 \mathrm{meV}$ in cohesive energy, see Table I), not only on a substrate (in experiments) but also when it is freestanding. So, in the rest of the paper, we will only concentrate on the $\mathrm{T}_{d}$ phase of the compound.

The elastic properties of a two-dimensional (2D) material can be characterized by two independent constants: the in-plane stiffness $C$, which represents the rigidity or the flexibility of the material, and the Poisson's ratio $\nu$, which is defined as the mechanical response of the material to applied external stress. Most of the materials have the tendency to compress in one direction when they are expanded in the perpendicular directions. This phenomenon is known as Poisson's effect. The ratio of the transverse contraction strain to longitudinal expansion strain is defined as the measure of this effect, namely, Poisson's ratio $\nu=-\epsilon_{\text {trans }} / \epsilon_{\text {axial }}$.

The elastic constants can be deduced from DFT calculations taking the relation between the total energy and the applied strain to be $E_{S}=c_{1} \varepsilon_{x}{ }^{2}+c_{2} \varepsilon_{y}{ }^{2}+c_{3} \varepsilon_{x} \varepsilon_{y}$ in the harmonic approximation, where $E_{S}$ is the energy difference between the strained and unstrained structures and $\varepsilon_{x}$ and $\varepsilon_{y}$ are the applied strain along the parallel and perpendicular directions to the dimers, respectively. The in-plane stiffness of the material along $x$ and $y$ directions are then defined as $C_{x}$ $=\left(1 / S_{0}\right)\left(2 c_{1}-c_{3}^{2} / 2 c_{2}\right)$ and $C_{y}=\left(1 / S_{0}\right)\left(2 c_{2}-c_{3}^{2} / 2 c_{1}\right)$, where $S_{0}$ is the unstretched area of the supercell, respectively. Similarly, the Poisson's ratio of the material along $x$ and $y$ directions are defined as $\nu_{x}=c_{3} / 2 c_{2}$ and $\nu_{y}=c_{3} / 2 c_{1}$, respectively. Hence, the elastic properties can be calculated if the values of $c_{1}, c_{2}$, and $c_{3}$ constants in the definition of $E_{S}$ are known.

In order to find these constants, we apply strain $\varepsilon_{x}$ (along dimer) and $\varepsilon_{y}$ (perpendicular to dimer) to the $4 \times 2 \times 1$ supercell of monolayer $\mathrm{T}_{d}-\mathrm{WTe}_{2}$ by changing the lattice constant from $-2 \%$ to $2 \%$ with steps of $1 \%$ along the $x$ and $y$ directions. We first change the $\varepsilon_{x}$ in the given range by taking $\varepsilon_{y}=0$ and then change $\varepsilon_{y}$ by taking $\varepsilon_{x}=0$ and fit the data to the parabola from the definition of $E_{S}$. After obtaining the values for $c_{1}$ and $c_{2}$, we apply equal strain simultaneously along $x$ and $y$ directions, i.e., $\varepsilon_{x}=\varepsilon_{y}$ and fit the data to $E_{S}$ to 
a)

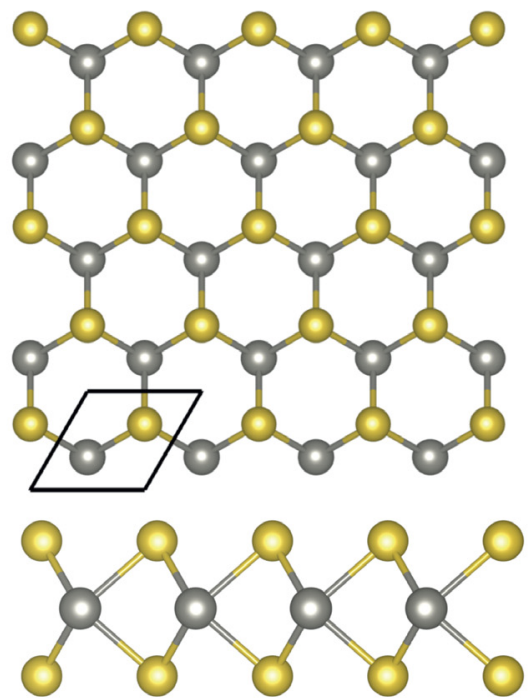

c)

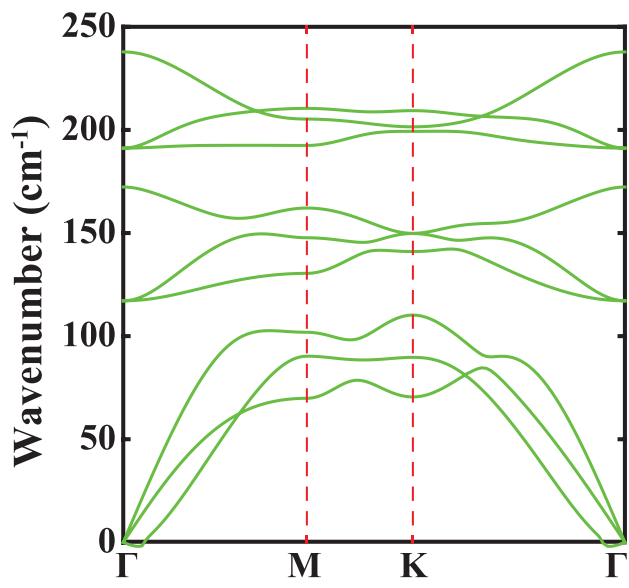

e)

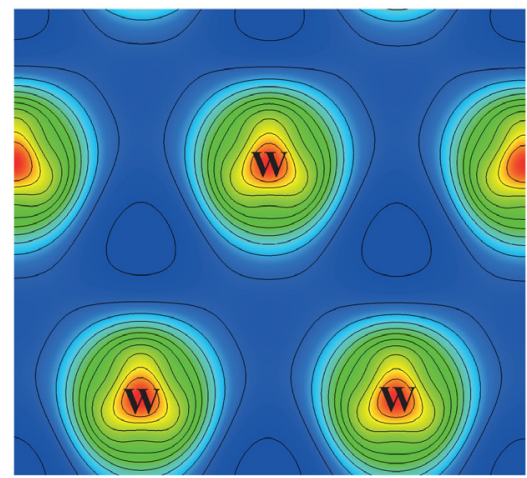

b)
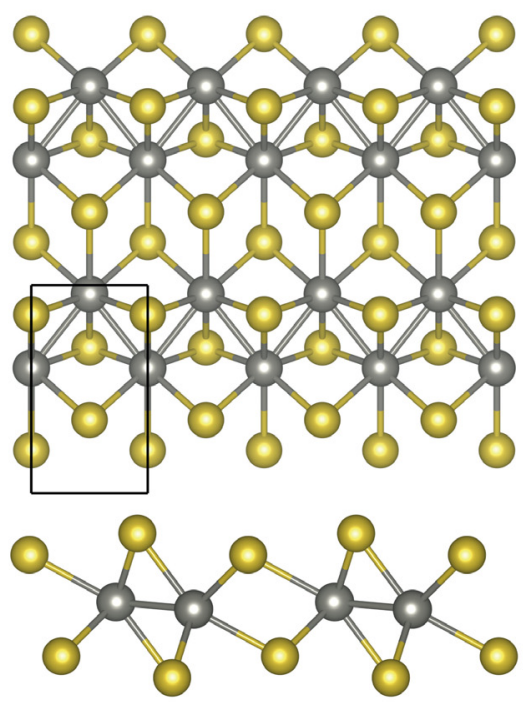

d)

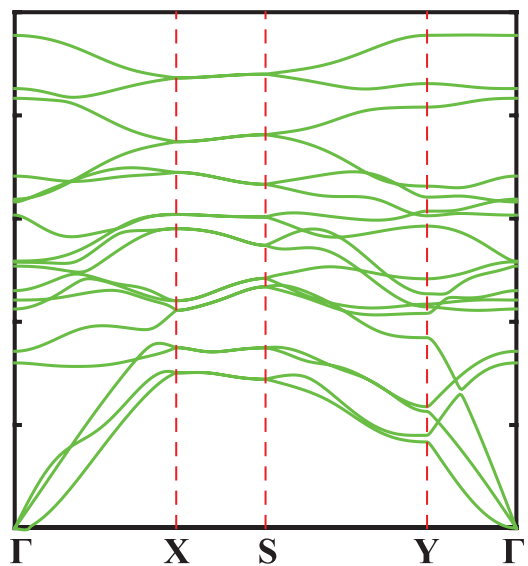

f)

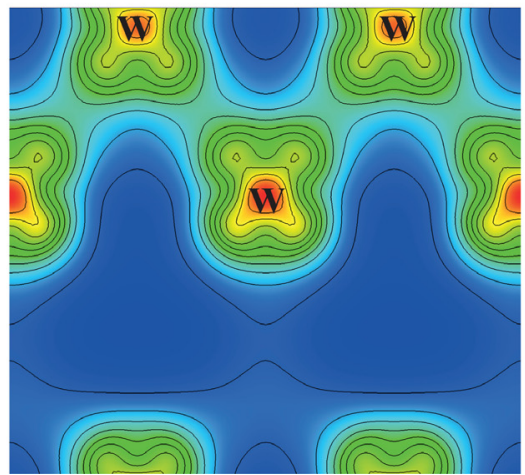

FIG. 1. Optimized geometric structure ((a) and (b)), phonon dispersion ((c) and (d)), and charge density contour plot ((f) and (g)) of $\mathrm{WTe}_{2}$ monolayer in respectively the $\mathrm{H}$ and $\mathrm{T}_{d}$ phases. The yellow and the grey atoms represent $\mathrm{Te}$ and $\mathrm{W}$ atoms, respectively. The hexagonal and the rectangular unit cells of $\mathrm{H}$ and $\mathrm{T}_{d}$ phases of $\mathrm{WTe}_{2}$ monolayer used in the calculations are shown in (a) and (b). The minimum, maximum isovalues, and the interval of contour lines are set to 0.08 (red), 0.00 (dark blue), and $0.01 \mathrm{e} / \AA^{3}$, respectively, in both charge density contour plots.

TABLE I. Calculated ground state properties of $\mathrm{T}_{d}$ (rectangular cell) and $\mathrm{H}$ phases (hexagonal cell) of WTe $\mathrm{F}_{2}$ monolayer. Calculated lattice parameters $a$ and $b$, the distance between two W atoms and W nearest neighbor Te atoms (the second nearest neighbor distance is given in the parentheses), the total amount of charge lost by the W atoms $\Delta \rho$, workfunction $\Phi$, calculated Poisson's ratio $\nu$ and in-plane stiffness along the W-W dimer direction $C$, the values for the perpendicular direction are given in the parentheses.

\begin{tabular}{|c|c|c|c|c|c|c|c|c|c|c|}
\hline & $a(\AA)$ & $b(\AA)$ & $d_{W-W}(\AA)$ & $d_{W-T e}(\AA)$ & $\Delta \rho(e)$ & $E_{c} /$ atom $(\mathrm{eV})$ & $E_{g}(\mathrm{eV})$ & $\Phi(\mathrm{eV})$ & $\nu$ & $C\left(\mathrm{eV} / \AA^{2}\right)$ \\
\hline $\mathrm{T}_{d}-\mathrm{WTe}_{2}$ & 3.50 & 6.30 & 2.85 & $2.73(2.83)$ & 0.50 & 4.57 & 0.00 & 4.39 & $0.26(0.38)$ & $4.45(6.56)$ \\
\hline $\mathrm{H}-\mathrm{WTe} \mathrm{e}_{2}$ & 3.55 & 3.55 & 3.55 & 2.73 & 0.53 & 4.54 & 0.75 & 4.45 & 0.18 (Ref. 35) & 5.42 (Ref. 35) \\
\hline
\end{tabular}


find $c_{3}$. In order to check the accuracy our method, we calculated the $C$ and the $\nu$ parameters of graphene and found as $21.42 \mathrm{eV} / \AA^{2}$ and 0.17 , respectively. These values are consistent with the earlier reported theoretical values which are $21.25 \mathrm{eV} / \AA^{2}$ and 0.16 , respectively, and shows the accuracy of our methodology. ${ }^{37,38}$ The experimental $\nu$ value is also consistent with the theoretical results which is $0.19 .{ }^{39}$

In isotropic 2D materials, the elastic constants along $x$ and $y$ directions are identical due to the symmetry of the lattice. Our calculations show anisotropy in the Poisson's ratio and the in-plane stiffness along $x$ (parallel to dimer) and $y$ (perpendicular to dimer) directions of the $\mathrm{T}_{d}-\mathrm{WTe}_{2}$ monolayer. This is expected since the dimerization breaks the symmetry of the structure. The calculated in-plane stiffness along parallel and perpendicular directions to the dimer are $4.45 \mathrm{eV} / \AA^{2}$ and $6.56 \mathrm{eV} / \AA^{2}$, respectively. These values are smaller than that of graphene $\left(21.42 \mathrm{eV} / \AA^{2}\right)$ and functionalized graphene-like materials. This indicates that $\mathrm{WTe}_{2}$ is more flexible than these materials. The calculated Poisson's ratio along the parallel and perpendicular directions to the dimers are also anisotropic, having values 0.26 and 0.38 , respectively. These values are slightly larger than that of graphene $(0.17)$ and its derivatives which indicates the stronger ability of preserving the equilibrium area of $\mathrm{WTe}_{2}$. The anisotropy in Poisson's ratio shows that the compound is less responsive under strain along the dimers than in the perpendicular direction.

As a further investigation, we also analyze the mechanical response of the compound under high strain values. For this purpose, we used a $2 \times 1$ supercell of $\mathrm{T}_{d}-\mathrm{WTe}_{2}$ monolayer and apply subsequently strain only parallel to the dimer direction and only perpendicular to the dimer direction. It was shown that applying negative stress (contracting) in the perpendicular direction to the $\mathrm{W}-\mathrm{W}$ dimers results in a transition from $\mathrm{T}_{d}$ to $\mathrm{H}$ structure in the $\mathrm{WTe}_{2}$ monolayer. ${ }^{40}$ However, here we apply only positive stress (pulling).

Our test calculations show that the Poisson's ratio and the in-plane stiffness values are almost the same for
$2 \times 1 \times 1$ and $4 \times 2 \times 1$ supercells. Stress versus strain curve of $\mathrm{T}_{d}-\mathrm{WTe}_{2}$ monolayer is presented in Fig. 2(a). Here, we define the in-plane stress as the derivative of energy (per unitcell) with respect to strain, divided by the width of the unitcell in the perpendicular direction of the applied stress. Unlike the 3D counterpart of stress that has units of pressure, the $2 \mathrm{D}$ in-plane stress has units of force. This is because in $3 \mathrm{D}$ case the derivative of energy with respect to strain is divided by the width and height in perpendicular direction while 2D crystals have no height.

The stress versus strain curves are qualitatively different when $\mathrm{T}_{d}-\mathrm{WTe}_{2}$ is pulled in the directions parallel and perpendicular to the $\mathrm{W}-\mathrm{W}$ dimers. In the perpendicular case, the curve reaches a maximum at the strain value of $\sim 15 \%$ and the structure suddenly ruptures as the brittle materials does. On the other hand, when the material is pulled parallel to the dimers, the structure continues to smoothly elongate passing the maximum point ( $\sim 11 \%$ strain) of the stress-strain curve acting like a ductile material. However, the instability that occurs after passing this maximum point can be clearly spotted in the phonon dispersions of the strained $\mathrm{T}_{d}-\mathrm{WTe}_{2}$. In Fig. 2(b), we plot the phonon dispersions of $\mathrm{T}_{d^{-}}-\mathrm{WTe}_{2}$ under $0.00,0.06$, and 0.12 strain in the parallel direction to the $\mathrm{W}-\mathrm{W}$ dimers. As the structure is strained, both optic and acoustic phonon modes soften. Above the maximum point of the stress-strain curve, one of the acoustic modes become imaginary at certain portion of the BZ indicating instability.

\section{Electronic and optical properties}

The band structure of $\mathrm{T}_{d}-\mathrm{WTe}_{2}$ monolayer is shown in Fig. 3(a). The compound has semimetallic ground state when it is unstrained. The valence and the conduction bands are crossing the Fermi level along the $\Gamma-\mathrm{X}$ and $\Gamma-\mathrm{Y}$ (very close to the $\Gamma$ point) directions in the BZ. The valence band maximum (VBM) of the monolayer is at the $\Gamma$ point, while the CBM is situated along the $\Gamma$-X direction but closer to the $\Gamma$ point. In the unstrained semimetallic case, the CBM is $6.7 \mathrm{meV}$ below the VBM (the first data point in Fig. 3(b)).
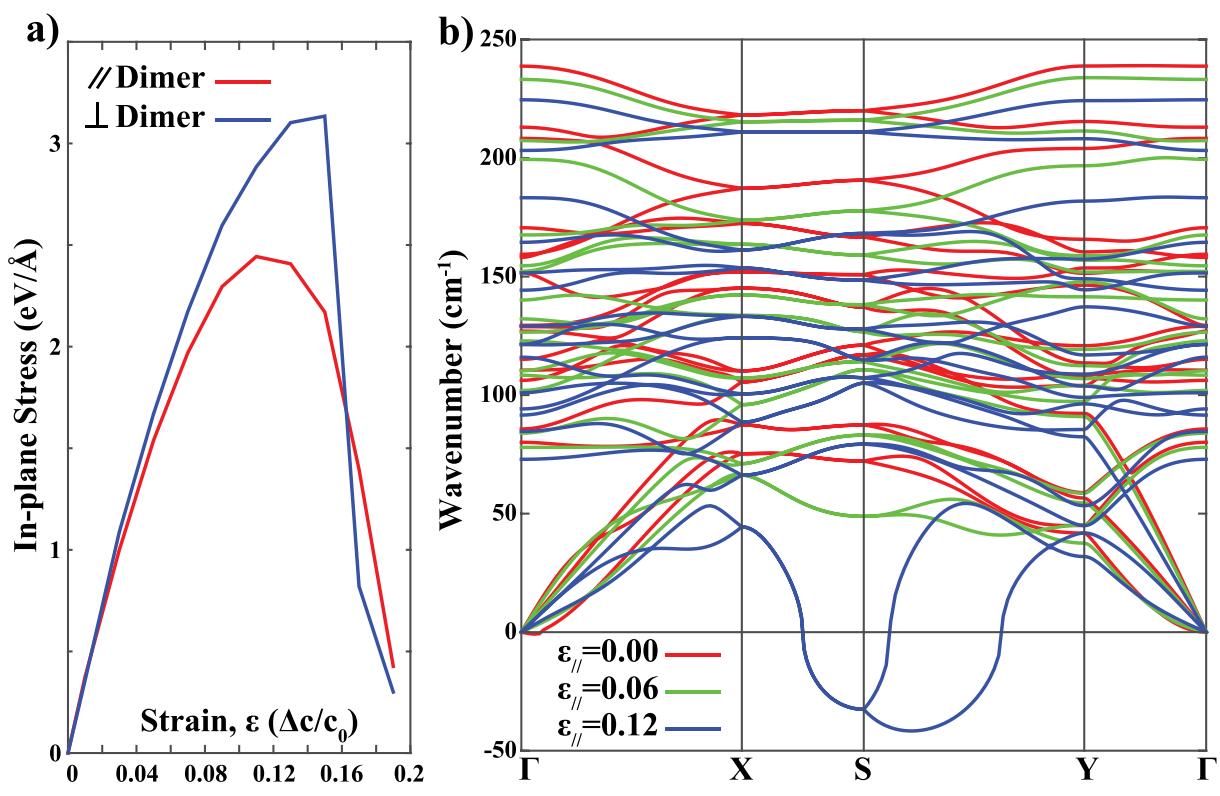

FIG. 2. (a) The stress versus strain curve of $\mathrm{T}_{d}-\mathrm{WTe}_{2}$. (b) The phonon dispersions of $\mathrm{T}_{d}-\mathrm{WTe}_{2}$ when there is no applied strain (red line) and 0.06 (green line) and 0.12 (blue line) strain parallel to the $\mathrm{W}-\mathrm{W}$ dimers. 


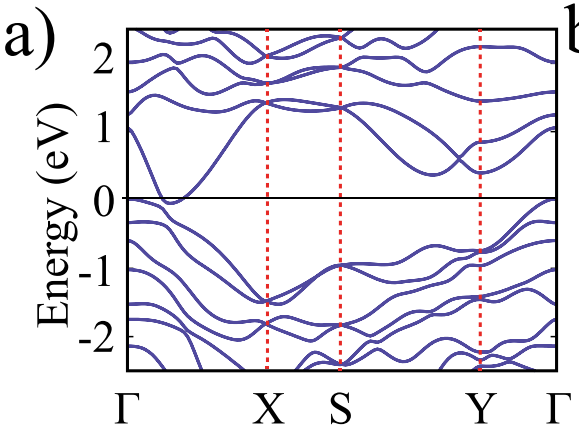

c)
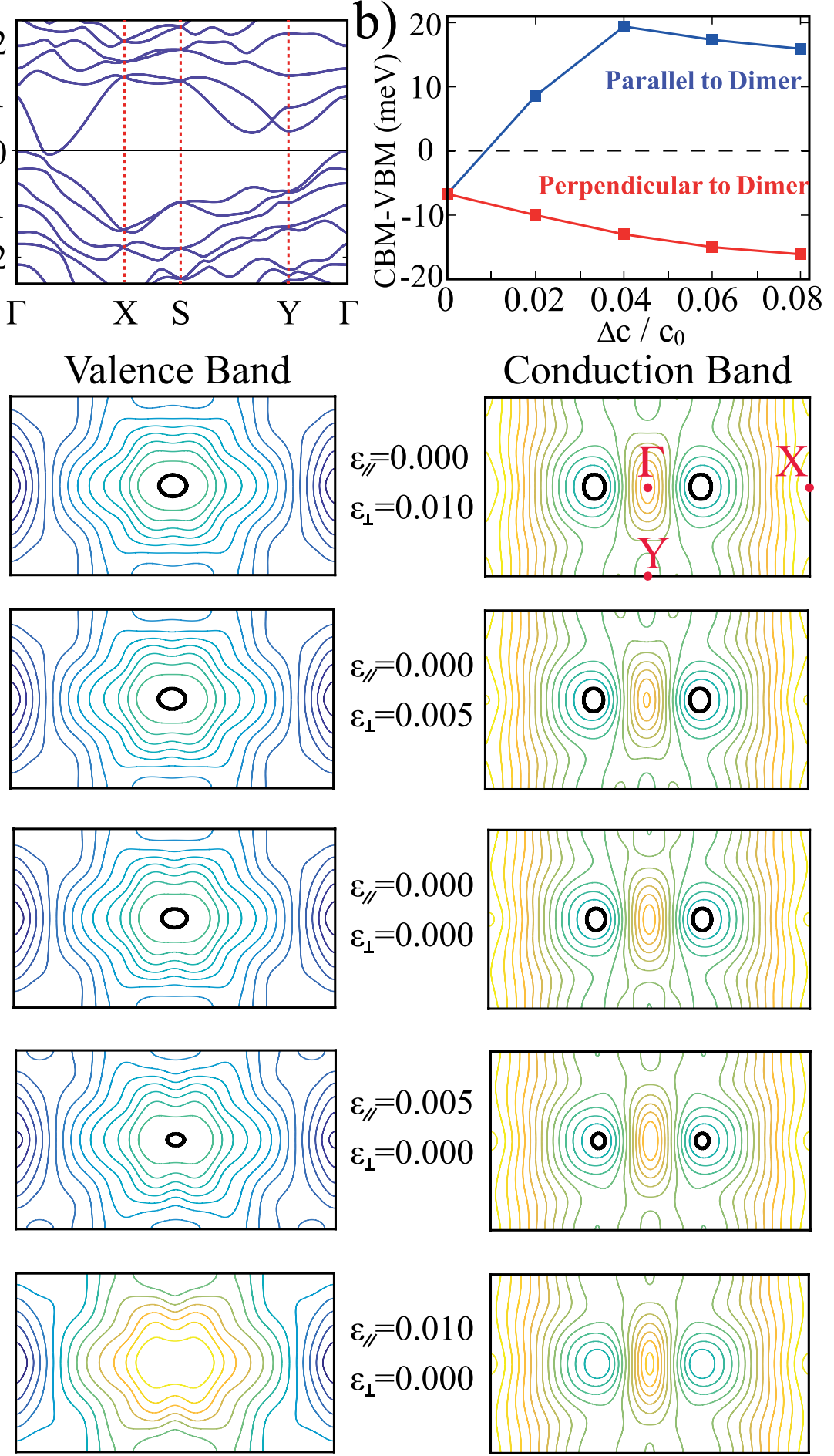

\section{Conduction Band}

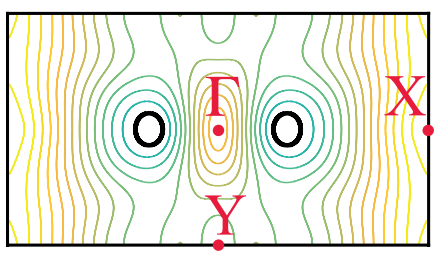

FIG. 3. (a) The electronic band structure of the $\mathrm{T}_{d}$ phase of monolayer $\mathrm{WTe}_{2}$, the Fermi level is set to $0 \mathrm{eV}$. (b) The energy difference between conduction band minimum (CBM) and the valence band maximum (VBM) of $\mathrm{T}_{d}-\mathrm{WTe}_{2}$ monolayer under applied external strain parallel and perpendicular directions to the $\mathrm{W}-\mathrm{W}$ dimers, $\mathrm{c}_{0}$ is the unstrained length of the lattice constants. The $x$ axis of the figure corresponds to the ratio of the difference between strained and unstrained lattice parameters $(\Delta c)$ to unstrained lattice parameters (c). (c) 2D contour plot of the valence and conduction band edges for various applied strains. The black lines correspond to the Fermi surfaces created by the valence and the conduction band edges crossing the Fermi level. The difference between the contour lines is set to $0.1 \mathrm{eV}$. The $\mathrm{x}$ and $\mathrm{y}$ directions correspond to parallel and perpendicular directions to the $\mathrm{W}-\mathrm{W}$ dimer.
The conduction band crosses the Fermi level along the $\Gamma-\mathrm{X}$ direction which is the $\mathrm{W}-\mathrm{W}$ dimer direction, in accordance with the fact that the dimerization contributes to the metallic ground state of the $\mathrm{T}_{d}-\mathrm{WTe} \mathrm{T}_{2}$ monolayer. In order to observe the effect of the reduction in the W-W interaction on the electronic structure, in Fig. 3(b), we plot the energy difference between the CBM and the VBM with respect to increasing applied tensile strain in the parallel and the perpendicular directions to the $\mathrm{W}-\mathrm{W}$ dimers. As mentioned before, the CBM is below the VBM in the unstrained phase. When the external tensile strain is applied along the W-W dimer, the CBM moves up while the VBM moves down in energy and the system undergoes a semimetal to semiconductor transition at $1 \%$ strain. On the other hand, when external strain is applied along the perpendicular direction to the $\mathrm{W}-\mathrm{W}$ dimers, the compound stays semimetallic even for large strain values. This prediction, together with its superconducting and anisotropic magnetoresistance properties, can be relevant when using $\mathrm{WTe}_{2}$ monolayers in nanoelectronic devices.

The anisotropic electronic structure of $\mathrm{T}_{d}-\mathrm{WTe} \mathrm{T}_{2}$ is even more clear when the conduction and valence band edges are presented as $2 \mathrm{D}$ contour plots along the whole BZ, as seen in Fig. 3(c). Here, the black circles correspond to the Fermi 


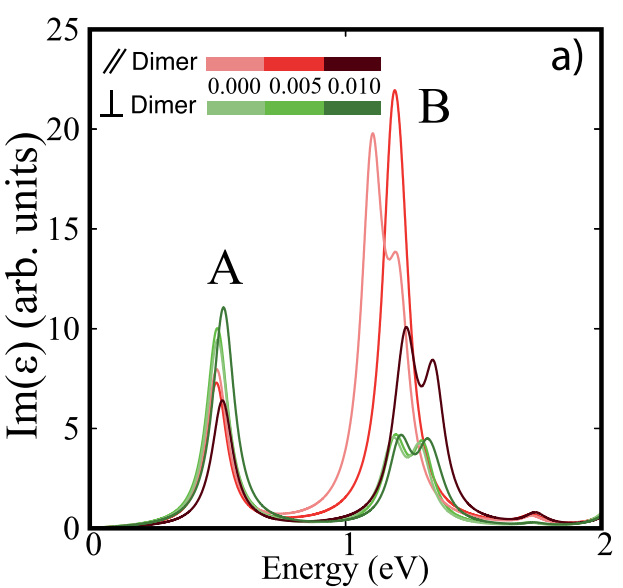

$\left.\right|_{2}$

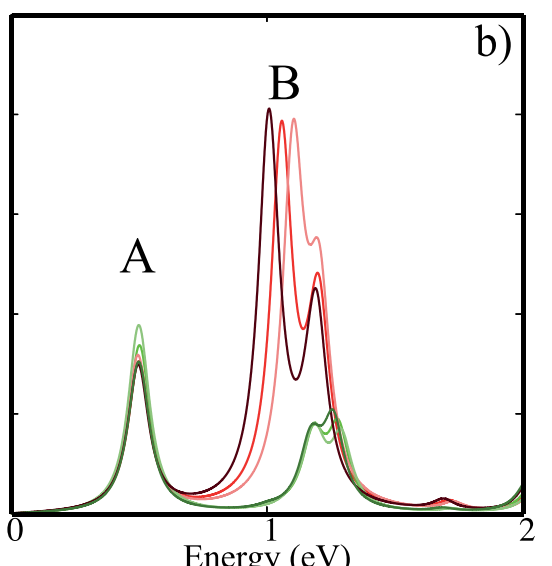

FIG. 4. Strain dependent imaginary part of the dielectric function of $\mathrm{T}_{d^{-}}$ $\mathrm{WTe}_{2}$ monolayer (a) when strain is along $\mathrm{W}-\mathrm{W}$ dimers and (b) perpendicular to W-W dimers. The light, normal, and dark red (green) curves corresponding to the dielectric function along (perpendicular) W-W direction under $0,0.005$, and 0.010 strain values, respectively. surfaces created by the valence and the conduction band edges both crossing the Fermi level. The surface created by the valence band edge is centered around the $\Gamma$ point, while the conduction band edge creates surfaces centered along the $\Gamma$-X direction (i.e., this is the X-axis in Fig. 3(c)). When tensile strain is applied along the perpendicular direction to the $\mathrm{W}-\mathrm{W}$ dimers, the contour plots exhibit minor changes. However, when the tensile strain is applied along the dimer direction the Fermi surfaces shrink and finally disappear. The semimetal to semiconductor transition occurs at approximately $1 \%$ strain.

In Fig. 4, we plot the strain dependent imaginary part of the dielectric function of $\mathrm{T}_{d}$-WTe 2 monolayer. Figs. 4(a) and 4 (b) represent the dependence of the dielectric function to strain along (red curves) and perpendicular (green curves) to the W-W dimers in the compound. The light, normal, and dark red (green) represent the dielectric function for different strain values of $0.000,0.005$, and 0.010 , respectively. As can be seen from the figures, due to the different symmetry along these two directions, the imaginary part of the dielectric function is different. The position of the $A$ peak is the same for both directions with and without external tensile strain; however, their intensity is not the same. The position of the peak $B$ is slightly different along the different directions for the unstrained case. The peak of the dielectric function along the dimer direction is closer to the peak $A$ than for the other direction. When 0.010 external tensile strain is applied along the dimers (Fig. 4(a)), peak $B$ shifts to higher energy and the peak position of the dielectric function along and perpendicular to the direction of the dimers becomes almost equal.

The reaction of the dielectric function to the external tensile strain applied along the perpendicular direction to the W-W dimers (Fig. 4(b)) are different from the previous case. As can be seen from the figure, the $B$ peak shifts to lower energies when strain is applied in the perpendicular direction to the dimers contrary to the case shown in Fig. 4(a). When strain reaches 0.010 , the peak for the dielectric function along and perpendicular to the dimer are separated from each other. Another interesting point is that the dielectric function for the perpendicular direction to the dimers is independent from the applied strain (green lines), its peak position do not change with external tensile strain.

\section{CONCLUSIONS}

In this work, we investigated the anisotropic mechanical, electronic, and optical properties of $\mathrm{T}_{d}-\mathrm{WTe}_{2}$ monolayer. We found that the $\mathrm{T}_{d}$ phase of the $\mathrm{WTe}_{2}$ monolayer, which exhibits $\mathrm{W}-\mathrm{W}$ dimerization along one of the lattice parameter, has $\sim 0.075 \mathrm{eV}$ lower energy per formula unit than the $\mathrm{H}$ phase. This $\mathrm{W}-\mathrm{W}$ dimerization changes the response of the compound to external tensile strain depending on the direction of the applied strain with respect to the dimerization direction. This leads to a different Poisson's ratio and different in-plane stiffness along and perpendicular direction of the $\mathrm{W}-\mathrm{W}$ dimers. Our strain dependent electronic structure calculations show that the $\mathrm{T}_{d}-\mathrm{WTe}_{2}$ monolayer becomes a semiconductor when it is strained by $1 \%$ along the dimer direction while strain along the perpendicular direction has minor effects on the electronic structure. Our investigations on the strain dependent optical properties of the compound show that the imaginary part of the dielectric function behaves differently along the different directions. Our calculations reveal that monolayer $\mathrm{WTe}_{2}$ together with its anisotropic and tunable properties may find applications in the field of nanoscale devices.

\section{ACKNOWLEDGMENTS}

This work was supported by the Flemish Science Foundation (FWO-Vl) and the Methusalem foundation of the Flemish government. Computational resources were provided by TUBITAK ULAKBIM, High Performance and Grid Computing Center (TR-Grid e-Infrastructure). H.S. was supported by a FWO Pegasus Long Marie Curie Fellowship. S.C. and A.R. acknowledge the financial support from the Marie Curie grant FP7-PEOPLE-2013-IEF Project No. 628876, European Research Council (ERC-2010-AdG267374), Spanish grant (FIS2013-46159-C3-1-P), Grupos Consolidados (IT578-13), and AFOSR Grant No. FA238615-1-0006 AOARD 144088, H2020-NMP-2014 project MOSTOPHOS, GA No. SEP-210187476, and COST Action MP1306 (EUSpec). S.C. acknowledges the support from The Scientific and Technological Research Council of Turkey (TUBITAK) under Project No. 115F388.

${ }^{1}$ Q. H. Wang, K. Kalantar-Zadeh, A. Kis, J. N. Coleman, and M. S. Strano, Nat. Nanotechnol. 7, 699 (2012). 
${ }^{2}$ B. Radisavljevic, A. Radenovic, J. Brivio, V. Giacometti, and A. Kis, Nat. Nanotechnol. 6, 147 (2011).

${ }^{3}$ M. Chhowalla, Nat. Chem. 5, 263 (2013).

${ }^{4}$ K. Mak, C. Lee, J. Hone, J. Shan, and T. F. Heinz, Phys. Rev. Lett. 105, 136805 (2010).

${ }^{5}$ A. Splendiani, Nano Lett. 10, 1271 (2010).

${ }^{6}$ S. Tongay, H. Sahin, C. Ko, A. Luce, W. Fan, K. Liu, J. Zhou, Y.-S. Huang, C.-H. Ho, J. Yan, D. F. Ogletree, S. Aloni, J. Ji, S. Li, J. Li, F. M. Peeters, and J. Wu, Nat. Commun. 5, 3252 (2014).

${ }^{7}$ S. Yang, C. Wang, H. Sahin, H. Chen, Y. Li, S.-S. Li, A. Suslu, F. M. Peeters, Q. Liu, J. Li, and S. Tongay, Nano Lett. 15, 1660 (2015).

${ }^{8}$ K. C. Santosh, C. Zhang, S. Hong, R. M. Wallace, and K. Cho, 2D Mater. 2, 035019 (2015)

${ }^{9}$ M. K. Jana, A. Singh, D. J. Late, C. Rajamathi, K. Biswas, C. Felser, U. V. Waghmare, and C. N. R. Rao, J. Phys.: Condens. Matter 27, 285401 (2015).

${ }^{10}$ B. E. Brown, Acta Cryst. 20, 268 (1966).

${ }^{11}$ W. G. Dawson and D. W. Bullett, J. Phys. C: Solid State Phys. 20, 6159 (1987).

${ }^{12}$ J. Augustin, V. Eyert, Th. Boker, W. Frentrup, H. Dwelk, C. Janowitz, and R. Manzke, Phys. Rev. B 62, 10812 (2000).

${ }^{13}$ M. N. Ali, J. Xiong, S. Flynn, J. Tao, Q. D. Gibson, L. M. Schoop, T. Liang, N. Haldolaarachchige, M. Hirschberger, N. P. Ong, and R. J. Cava, Nature 514, 205 (2014).

${ }^{14}$ I. Pletikosić, M. N. Ali, A. V. Fedorov, R. J. Cava, and T. Valla, Phys. Rev. Lett. 113, 216601 (2014).

${ }^{15}$ Z. Zhu, X. Lin, J. Liu, B. Fauqué, Q. Tao, C. Yang, Y. Shi, and K. Behnia, Phys. Rev. Lett. 114, 176601 (2015).

${ }^{16}$ L. R. Thoutam, Y. L. Wang, Z. L. Xiao, S. Das, A. Luican-Mayer, R. Divan, G. W. Crabtree, and W. K. Kwok, Phys. Rev. Lett. 115, 046602 (2015).

${ }^{17}$ X.-C. Pan, X. Chen, H. Liu, Y. Feng, Z. Wei, Y. Zhou, Z. Chi, L. Pi, F. Yen, F. Song, X. Wan, Z. Yang, B. Wang, G. Wang, and Y. Zhang, Nat. Commun. 6, 7805 (2015).

${ }^{18}$ P. S. Alekseev, A. P. Dmitriev, I. V. Gornyi, V. Yu. Kachorovskii, B. N. Narozhny, M. Schütt, and M. Titov, Phys. Rev. Lett. 114, 156601 (2015).

${ }^{19}$ P. L. Cai, J. Hu, L. P. He, J. Pan, X. C. Hong, Z. Zhang, J. Zhang, J. Wei, Z. Q. Mao, and S. Y. Li, Phys. Rev. Lett. 115, 057202 (2015).
${ }^{20}$ D. Kang, Y. Zhou, W. Yi, C. Yang, J. Guo, Y. Shi, S. Zhang, Z. Wang, C. Zhang, S. Jiang, A. Li, K. Yang, Q. Wu, G. Zhang, L. Sun, and Z. Zhao, Nat. Commun. 6, 7804 (2015).

${ }^{21}$ W.-D. Kong, S.-F. Wu, P. Richard, C.-S. Lian, J.-T. Wang, C.-L. Yang, Y.-G. Shi, and H. Ding, Appl. Phys. Lett. 106, 081906 (2015).

${ }^{22}$ Y. Zhao, H. Liu, J. Yan, W. An, J. Liu, X. Zhang, H. Jiang, Q. Li, Y. Wang, X. Z. Li, D. Mandrus, X. C. Xie, M. Pan, and J. Wang, Phys. Rev. B 92, 041104(R) (2015).

${ }^{23}$ F. X. Xiang, M. Veldhorst, S. X. Dou, and X. L. Wang, e-print arXiv:1504.01460

${ }^{24}$ Y. M. Dai, J. Bowlan, H. Li, H. Miao, Y. G. Shi, S. A. Trugman, J.-X. Zhu, H. Ding, A. J. Taylor, D. A. Yarotski, and R. P. Prasankumar, Phys. Rev. B 92, 161104(R) (2016).

${ }^{25}$ Y. Luo, H. Li, Y. M. Dai, H. Miao, Y. G. Shi, H. Ding, A. J. Taylor, D. A. Yarotski, R. P. Prasankumar, and J. D. Thompson, Appl. Phys. Lett. 107, $182411(2015)$

${ }^{26}$ J. Jiang, F. Tang, X. C. Pan, H. M. Liu, X. H. Niu, Y. X. Wang, D. F. Xu, H. F. Yang, B. P. Xie, F. Q. Song, X. G. Wan, and D. L. Feng, Phys. Rev. Lett. 115, 166601 (2015).

${ }^{27}$ C. Ataca, H. Sahin, and S. Ciraci, J. Phys. Chem. C 116, 8983 (2012).

${ }^{28}$ P. Blöchl, Phys. Rev. B 50, 17953 (1994).

${ }^{29}$ G. Kresse and D. Joubert, Phys. Rev. B 59, 1758 (1999).

${ }^{30}$ G. Kresse and J. Hafner, Phys. Rev. B 47, 558(R) (1993).

${ }^{31}$ G. Kresse and J. Furthmüller, Phys. Rev. B 54, 11169 (1996).

${ }^{32}$ J. P. Perdew, K. Burke, and M. Ernzerhof, Phys. Rev. Lett. 77, 3865 (1996).

${ }^{33}$ G. Henkelman, A. Arnaldsson, and H. Jónsson, Comput. Mater. Sci. 36, 354 (2006).

${ }^{34}$ Y. C. Jiang and J. Gao, Scientific Reports 6, 19624 (2016).

${ }^{35}$ J. Kang, S. Tongay, J. Zhou, J. Li, and J. Wu, Appl. Phys. Lett. 102, 012111 (2013).

${ }^{36}$ D. Alfe, Comput. Phys. Commun. 180, 2622 (2009).

${ }^{37}$ F. Liu, P. Ming, and J. Li, Phys. Rev. B 76, 064120 (2007).

${ }^{38}$ K. N. Kudin, G. E. Scuseria, and B. I. Yakobson, Phys. Rev. B 64, 235406 (2001).

${ }^{39}$ A. Politano and G. Chiarello, Nano Res. 8, 1847 (2015).

${ }^{40}$ K.-A. N. Duerloo, Y. Li, and E. J. Reed, Nat. Commun. 5, 4214 (2014). 